\title{
Tracking Cataract by the Four-Line Method
}

\author{
K. J. Hanna* L. Tarassenko \\ Robotics Research Group \& Medical Engineering Unit \\ Department of Engineering Science \\ University of Oxford
}

An implementation of Scott's 'four-line' algorithm for estimating optic flow is presented. The application is the analysis of cataract motion in the human eye lens. Images are recorded using retro-illumination photography. Successive images recorded at up to a 6 month interval are used as the source for the flow recovery procedure. The four-line algorithm itself uses edge data from these images at several scales. A procedure for performing image registration and optic flow recovery using the same four-line algorithm is presented. Several examples of the algorithm output are given.

\section{INTRODUCTION}

This paper presents the results of an implementation of Scott's four-line method for recovering optic flow ${ }^{1}$. Edge data at several scales derived from the Canny algorithm ${ }^{2}$ are used as the image features to be tracked. The flow recovery procedure was performed on a sequence of in vivo cataract images recorded using retro-illumination photography.

Section 2 gives a brief medical background to cataract in relation to this paper so that the results of the algorithms may be interpreted. Section 3 discusses particular experimental and clinical constraints associated with the application, and shows that edge data at several scales are suitable features for the tracking procedure. Section 4 briefly discusses the basis of the four-line method and some other optic flow recovery algorithms. It is shown that an algorithm based on the four-line technique is suited to this application. Section 5 summarises the four-line technique, and section 6 describes the algorithms used for tracking cataract. The complete tracking procedure is then summarised in section 7. Several examples of the tracking algorithms are then presented in section 8 .

\section{MEDICAL BACKGROUND}

It is known that certain types of motion are associated with certain types of cataract ${ }^{3}$. Much information about the progression of the disease could be gained if cataract

\footnotetext{
*The author acknowledges the support of the Science and Engineering Research Council.
}

regions could be tracked over a time period. This is particularly relevant in relation to drug therapy, in which the efficacy of a therapeutic agent must be quantified.

Images are initially recorded on photographic film using retro-illumination photography ${ }^{4}{ }^{5}$. The technique produces an en face view of the human lens by the reflection of light from the optic disc through the human lens and onto a film plane. Figure 1 gives a typical example of the resultant image. The large, circular region in the centre of the image is the human lens. The dark region surrounding the lens is the iris. Any cataract regions in the lens results in a reduced light return at that point, leading to the dark regions within the lens shown in figure 1. It is these individual cataract regions which are to be tracked using successive retro-illumination images taken at up to 6 month intervals.

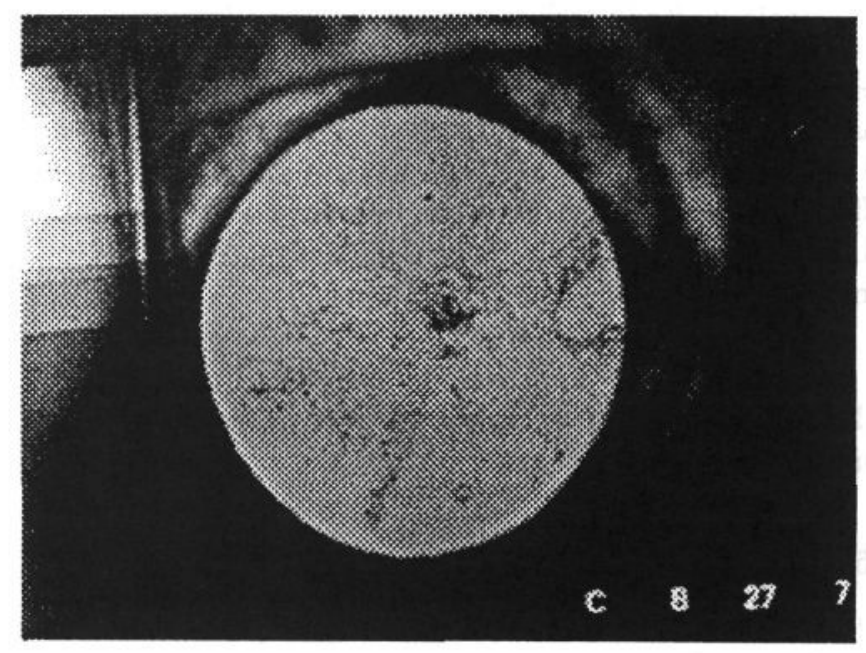

Figure 1: Retro-illumination image of a human lens

\section{EXPERIMENTAL AND CLINICAL CONSTRAINTS}

Due to experimental variation, the angular and translational position of the eye lens varies between images within a sequence. Therefore, an exact lens position is unknown from a priori information, leading to a registration problem when analysing a sequence of images. Also, a secondary effect is the slight motion effect due to image foreshortening. The difficulty here is the separation of motion due to experimental variation and that due to 
true cataract motion. The same experimental variation can cause changes in the global image intensity due to increased or decreased reflection from the optic disc. A final variation is the introduction of low frequency spatial gradients within the image due to the addition of light reflected from the cornea of the eye.

A clinical constraint which may be imposed is a bound on the maximum magnitude of the motion of cataract. Although cataract regions are known to move, the motion is 'small' in relation to the spatial extent of the complete cataract area for the time intervals involved. On a global scale, this constraint leads to the gross shape of the complete cataract area being maintained within an image sequence.

A final constraint is that motion boundaries describe the change in cataract. At this stage, we are primarily interested in the movement and spreading of smaller regions of cataract within larger lens areas.

\section{MOTION ALGORITHMS}

Several techniques may be employed to generate the motion field. Individual cataract regions may be segmented and matched using minimal mapping theory ${ }^{6}$. However, algorithms based on this technique are best suited for isolated cataract features. In many cases, cataracts are densely clustered within single regions (see figure 1).

Algorithms founded on the continuity and smoothness of motion ${ }^{78}$ could be used to recover the flow field. However, it was shown in the previous section that motion boundaries describe the change in cataract. Imposing the two constraints of continuity and smoothness of the flow field will distort the true flow field at these motion boundaries, leading to inaccurate estimates of the true motion.

An alternative approach is the four-line technique. This procedure is accurate in recovering a flow-field undistorted at boundaries.

\section{THE FOUR-LINE METHOD}

The method is described fully elsewhere ${ }^{1}$, however, a brief outline of the procedure is given below.

The first step in the algorithm is the calculation of a matching strength surface between features in a time series of images. Scott's algorithm employed a correlation technique for this purpose. The second step in the algorithm is to perform a principal axes decomposition within the match surface for every point in one image. The result is two orthogonal match constraint lines, each with an associated variance attached to it. This variance is a measure of the spread of possible matching points. These two constraint lines now remain fixed throughout the procedure. A second set of orthogonal constraint lines are then calculated for every point, based on the estimates of velocity of neighbouring points. In the first iteration of the algorithm, the initial estimates of the velocity field may be set to arbitrary values, or alternatively, estimates based on the matching surface can be chosen. A weighted least squares calculation is performed between the variances of the neighbour constraint lines and the match constraint lines described above. This results in a new estimate of velocity at each point. A set of new constraint lines may now be calculated and the process repeated until the new estimates of velocity are not significantly different from the previously calculated values.

The presence of two orthogonal match constraint lines at a point, each with low variance, suggests that point motion has occurred. However, if one match constraint line has a high variance, this suggests that edge motion has occurred. The procedure is effective at combining different smoothness assumptions depending on the different types of data structure. The resultant flow-field is accurate and undistorted at motion boundaries.

\section{THE CATARACT MOTION RE- COVERY PROCEDURE}

\section{Feature selection}

The four-line procedure requires the calculation of a matching strength surface between image features. It was shown in section 3 that absolute image intensities may not be used as a basis for this calculation. This suggests the use of edge data as a source for the motion recovery procedure.

It was also mentioned that the gross shape of the complete cataract is maintained over the time periods involved. These two points suggest a cataract motion recovery procedure which uses edge data at low scales to register the images, and edge data at higher scales to recover the true motion of individual cataract regions.

Edge data derived from the Canny algorithm ${ }^{2}$ was used in this implementation. The choice of edge scale is chosen by the adjustment of the Gaussian smoothing operator, which is the first step in the algorithm. Figure 3 gives examples of the algorithm output when applied to the lens regions corresponding to figure 2 . Subsets of the complete lens regions have been taken for illustrative purposes only. The edge data has been superimposed such that black indicates an edge due to the first image, and grey an edge due to the second image. Each image is from the same patient, but separated by a six month interval. The edge detection has been performed at a low spatial scale $(\sigma=12)$. Figure $2 \mathrm{c}$ shows the results of edge detection at a higher scale $(\sigma=4)$.

The Canny algorithm returns data on edgel position, strength and orientation. Edgel orientation is used as the basis of the calculation of the matching strength surface. 


\section{Recovering match constraint lines}

The first step in the four-line procedure is the recovery of the two orthogonal match constraint lines described in section 5 .

One of the first problems to be tackled is the choice of effective search area for a matching process. As mentioned in the previous section, the complete motion recovery procedure will result in large motion fields at low scales, and relatively small motion fields at higher scales. This may suggest a hierarchy of search areas, ranging from the complete image area at gross scales to tens of pixels at high scales. However, the selection of search area will be completely arbitrary and extremely image dependent.

A further point is the implied separation of image registration from true cataract motion in the procedure described above. This suggests that complete registration must be declared at one particular scale. Any motion recovered at higher scales would then be due to true cataract motion. However, the choice of such a scale would also be arbitrary and image dependent.

A partial solution to the problems described above is to use precisely the same algorithm at both low and high scales. Also, a technique can be devised for performing continuous registration at both low and high scales. Gross registration will still be performed at the lower scales, although a small registration vector may be returned at the higher scales.

\section{Choosing candidate matches}

It was shown in the previous section that a matching procedure must be devised which can operate at both low and high scales. One of the simplest adaptive procedures is the choice of the nearest edgels with similar orientations as candidate matches. However, a problem here occurs when part of a real edge within the cataract fails to be detected in both images of the sequence. The procedure could then choose incorrect candidate edgels from elsewhere in the image. Also, because the effective search space for the process must extend to the whole image, a small number of grossly incorrect matches could alter the resultant flow-field dramatically.

The converse problem occurs when edgels of similar orientation are declared as candidate matches when the true correspondence is further away. The effect of this type of incorrect match may not be as dramatic as the type described above, however large numbers of incorrect matches will result in an inaccurate estimate of the motion field.

\section{Reducing match errors}

The first of the two problems described above is addressed by performing an inverse matching procedure. A related technique was implemented by Spacek ${ }^{9}$.
Once the nearest candidate edgel in the second image has been chosen, the same procedure is performed between that edgel and the first image. If the nearest candidate edgel in the first image is in close proximity to the original point, then the original mapping is declared to be valid. If the two points are not in close proximity, then the original point is eradicated from further analysis.

The choice of threshold for the proximity measure is chosen by estimating the size of the smallest cataract feature to be tracked. The inverse tracking procedure is primarily designed to prevent candidate matches between edgels from completely unrelated cataract features. A threshold of 10 pixels was used in the implementation presented here.

As mentioned above, the edge points which do not satisfy the inverse tracking procedure are not analysed further in the algorithm presented here. In this application, it is more important to have a moderate number of accurate match selections than a larger number of grossly inaccurate match estimates. Qualitatively however, this has not led to a dramatic reduction in edge data for the four-line algorithm (see section 8).

As discussed above, the converse problem can occur when edgels of similar orientation are declared as candidate matches when the true correspondence is further away. Inaccurate match estimates due to this problem cannot be avoided using the matching procedure described above. However, the problem reduces as the cataract images are brought further into registration using the multi-scale procedure described in section 7 .

\section{Calculating matching strengths}

The procedure described above has chosen a single edgel as a candidate match. The inverse tracking technique has increased the likelihood that the candidate edgel is due to part of the correct cataract feature. A simple technique for calculating the match strength directly from the edge data is to analyse the spatial variance of edgels with similar orientations surrounding the candidate edgel. A principal axes decomposition can be used to derive the orientation of the match constraint lines. Calculation of the second moments of area with respect to each of these constraint lines gives a measure of the spread or variance about the candidate edgel. A low value for both moment areas indicates point motion. A high value in one direction with a low value in the other indicates edge motion.

These second moments of area can be used in heuristic formulae to return matching strengths for the four-line procedure.

\section{Neighbour selection}

The second part of the four-line procedure requires the selection of neighbour motion estimates. Analysing the surrounding eight pixels will usually return only two 
neighbour motion estimates from adjacent edgels within a linked collection of edgels. Choosing neighbour estimates by this technique prevents constraint propagation to other linked collections of edgels. A simple selection technique which allows such propagation is the choice of the nearest neighbour motion estimate in each of the surrounding four quadrants.

\section{Estimating registration vectors}

It was mentioned in section 6 that image registration and true cataract motion should not be separated. Therefore, once an estimate of the velocity field has been calculated using the four-line algorithm at any scale, it must always be described by rotational and translational registration vectors. A current solution is to rotate the velocity field synthetically until the variance of the new velocity field is minimised. The remaining velocity field is averaged and used as an estimate of the translation vector. Horn ${ }^{10}$ describes similar techniques for calculating rotational and translational components of a vector field.

\section{SUMMARY OF PROCEDURE}

The Canny algorithm is used to produce edge data at a large range of scales from a pair of successive retroillumination images. Automatic lens/iris segmentation is then performed by separate algorithms not described here.

Beginning with the lowest scale, the four-line procedure described in the previous sections is used to estimate the flow-field between the two images. Translational and rotational vectors are then recovered and used to bring all the edge data at every scale into closer registration. This procedure is repeated until the resultant translation and registration vectors are negligible.

The next highest scale is now selected and the complete procedure described above is repeated for all subsequent scales. The residual flow-field calculated at the highest scale is deemed to be due to cataract motion within the eye lens.

\section{RESULTS}

Two image sequences are presented. The first set of results is from retro-illumination images taken during the same day (Figure 2). Subsets of complete images have been taken for illustrative purposes only. Changes due to experimental variation discussed in section 3 will still occur, however there should be no motion due to cataract change.

Figure 3 shows superimposed Canny edge data at a low scale $(\sigma=12)$ from both images. Figure 4 shows the inverse tracking match vectors described in section 6 . The dark points indicate edge points with a valid candidate match in the second image. The grey points represent the velocity vector end, and the lighter points represent the vectors themselves. Figure 5 shows the result of the

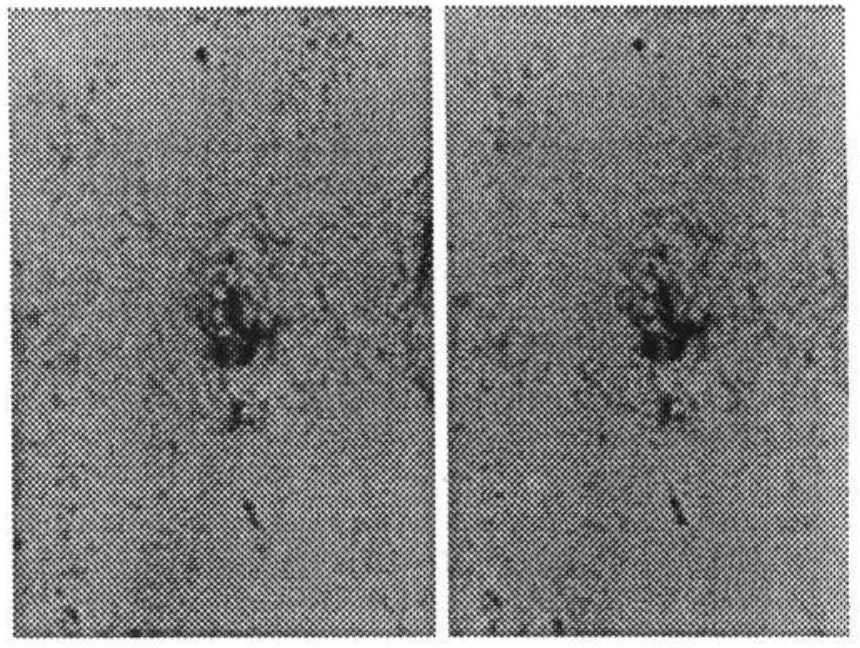

Figure 2: Subsets of images recorded on same day

four-line algorithm. Figure 6 shows the same Canny edge data superimposed after the images have been brought into closer registration using the technique described in section 6 . The edge data at this scale are brought completely into registration after the last rotation and translation procedure is performed.

Figure 7 shows superimposed Canny edge data at a high scale $(\sigma=4)$ after the registration procedure has been performed at the lower scale. Figure 8 shows the result of the four-line algorithm. If the calculated vectors are compared to figure 7 , it can be seen that the calculated motion field is in close correspondence to the actual motion of well-defined features within the edge data.

The edge data are brought into complete registration at this higher scale by further rotational and translational procedures described in section 6 .

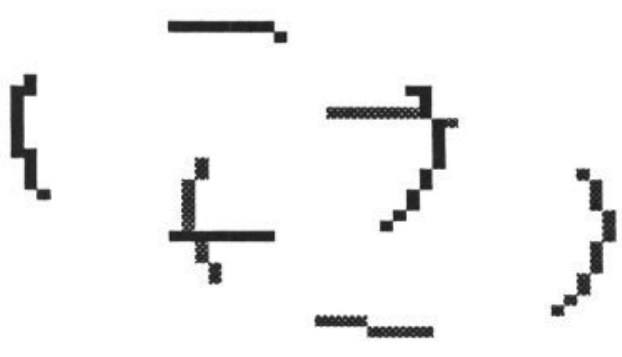

Figure 3: Superimposed edge data, $\sigma=12$ (lens subsets)

Figure 9 shows two subsets of cataract images taken from the same patient at a 6 month interval. Figure 10 on the left shows superimposed Canny edge data at a low scale $(\sigma=8)$ from both images after several registration procedures. Figure 10 on the right shows the final result of the four-line algorithm at this scale.

Figure 11 on the left shows superimposed Canny edge data at a higher scale $(\sigma=6)$ after the complete registration procedure has been performed at the lower scale. 


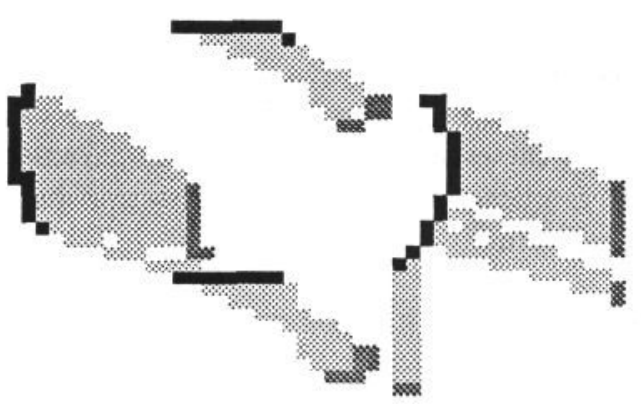

Figure 4: Match velocity estimates

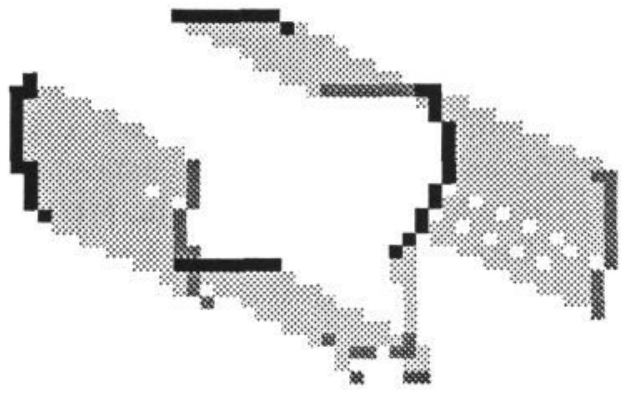

Figure 5: Calculated velocity field

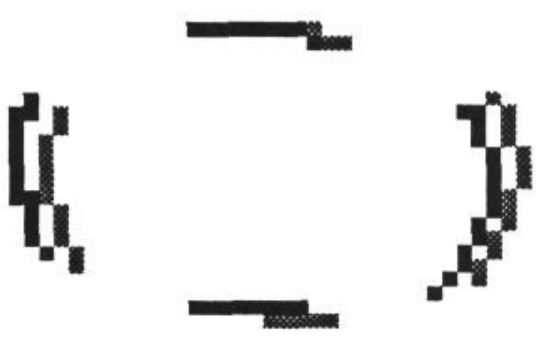

Figure 6: Superimposed edge data $(\sigma=12)$ after registration (lens subsets)

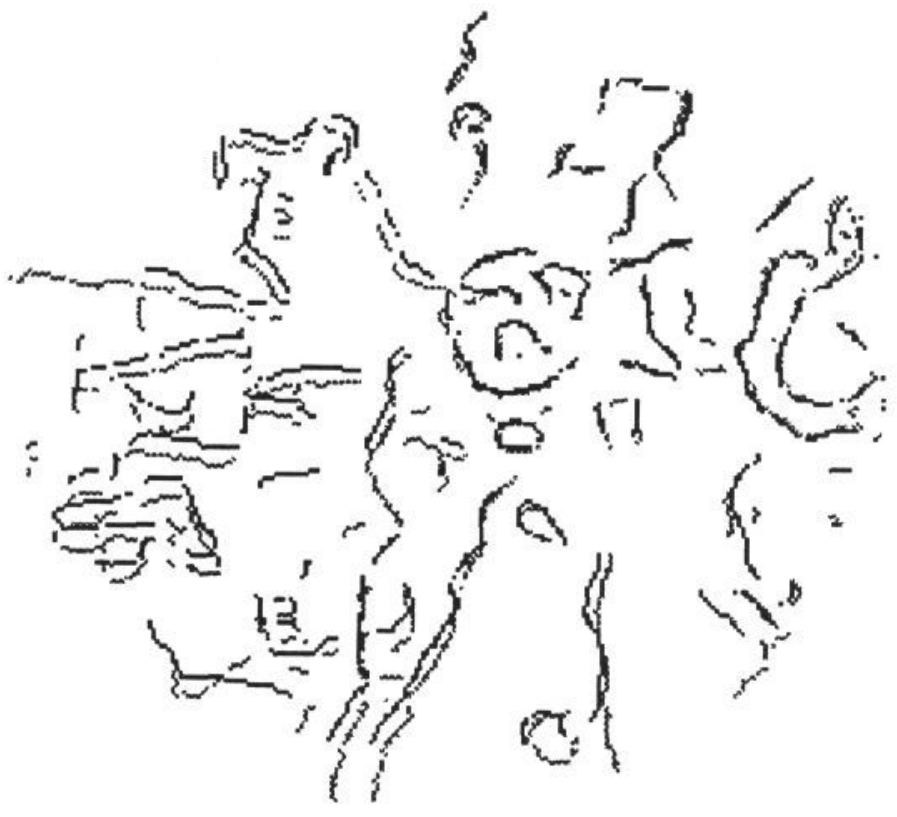

Figure 7: Superimposed edge data $(\sigma=4)$ after registration at a lower scale (complete lens)

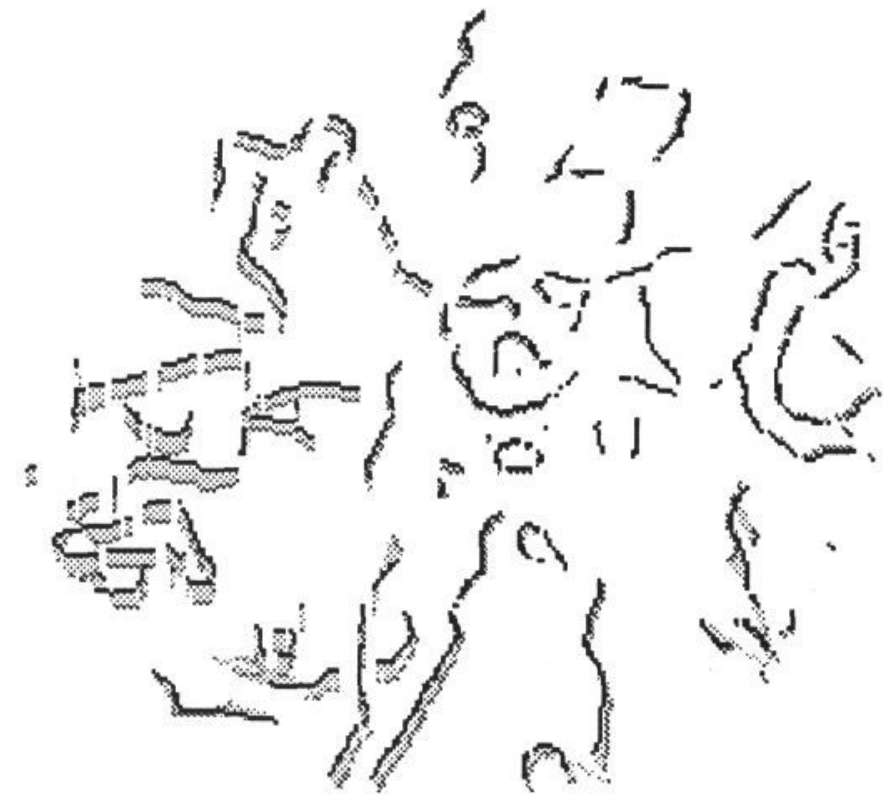

Figure 8: Calculated velocity field (complete lens)

Figure 11 on the right shows the result of the four-line algorithm procedure. Qualitatively, the flow-field appears to be converging in one direction, indicating the motion of cataract regions.
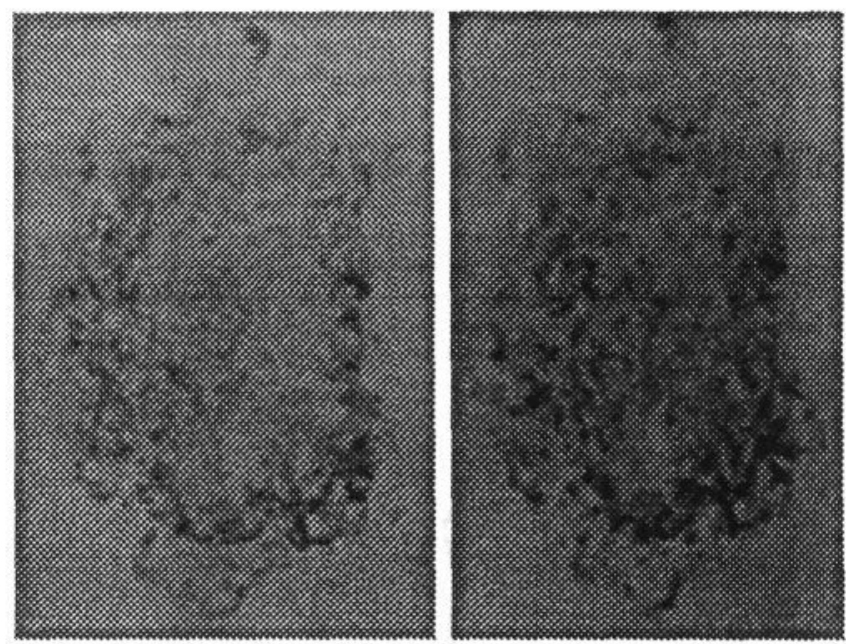

Figure 9: Subsets of images taken at a 6 month interval

\section{CONCLUSION}

The cataract tracking procedure described above has produced encouraging preliminary results on a small number of image sequences. The procedure based on the fourline technique performs registration and can track welldefined cataract regions at higher scales.

However, the implementation in its present form has several limitations. Firstly, only well-defined cataract regions can be tracked successfully. Regions which have greatly changed shape or size between successive images are less likely to be tracked. Secondly, the procedure at present does not compensate for the very slight motion 

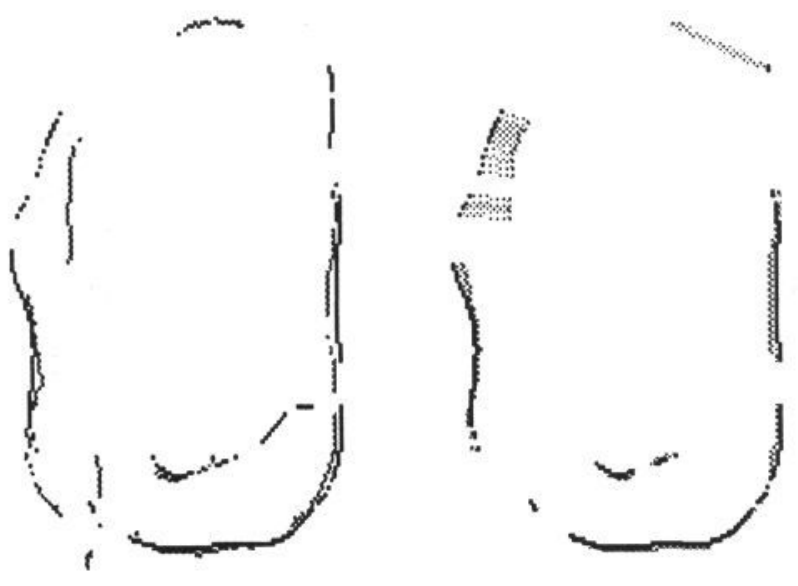

Figure 10: Superimposed edge data $(\sigma=8)$ and calculated flow-field

effects due to image foreshortening which occur due to variations in the eye position. A third limitation of the four-line algorithm in particular is the use of heuristic formulae in the estimation of weighting parameters. The parameters chosen in this implementation have limited theoretical value.

Notwithstanding this, the results have produced preliminary data which were previously unavailable to the clinician.

\section{ACKNOWLEDGEMENTS}

We would like to thank Guy Scott for our invaluable conversations concerning the implementation of his four-line motion algorithm. We would also like to thank Professor J.M. Brady for his enthusiasm and guidance in this work. Mr. A. J. Bron, Mr. G. A. Shun-Shin, Mr. J. Sparrow and Mr. N. Brown from the Oxford Eye Hospital have all provided invaluable clinical information.

\section{REFERENCES}

1. Scott G.L. Local and global interpretation of moving imagery PhD thesis, University of Sussex, UK (1986)

2. Canny J. "Finding Edges and Lines in Images" $M I T$ AI Laboratory Technical Report No. AI-TR-720 (1983)

3. Bron A.J., N. Brown, J. Sparrow, G.A. Shun-Shin "Medical Treatment of Cataract" Eye Vol 1 (1987) pp 542-550

4. Kawara, T., H. Obazawa, R. Nakano, M. Sasaki, T. Sakati "Quantitative evaluation of cataractous lens opacities with retro-illumination photography" $J p n$. J. Clin. Ophthalmol. Vol 33 (1979) pp 21-26
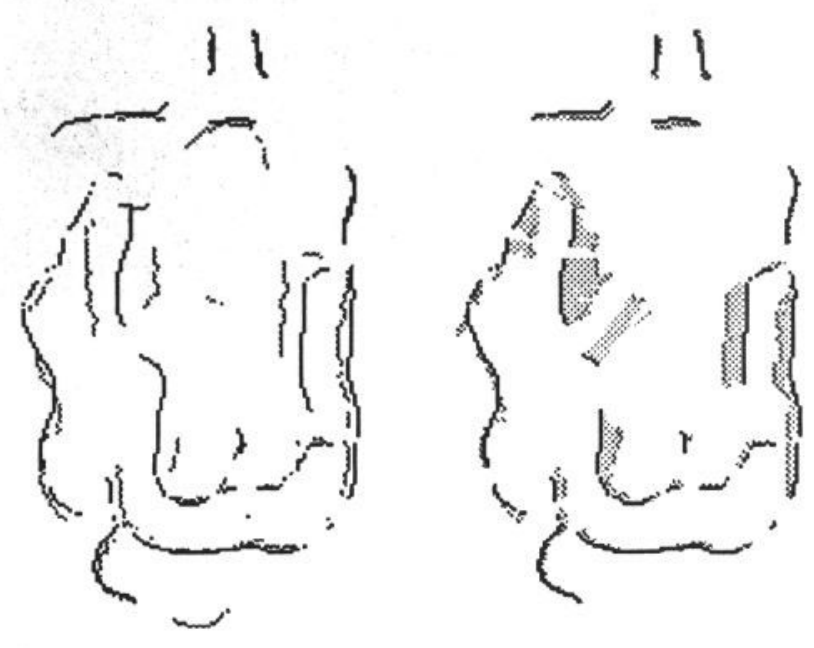

Figure 11: Superimposed edge data $(\sigma=6)$ and calculated flow-field
5. Kawara, T., H. Obazawa "A new method for retroillumination photography of cataractous lens opacities" Am. J. Ophthalmol. Vol 90 (1980) pp 186-189

6. Ullman S. The Interpretation of Visual Motion MIT Press, Cambridge, MA, USA (1979)

7. Horn B.K.P, B.G. Schunck "Determining Optical Flow" Artificial Intelligence Vol 17 (1981) pp 185203

8. Hildreth, E.C. The measurement of Visual Motion MIT Press, Cambridge, MA, USA (1984)

9. Spacek, L.A. The Detection of Contours and their Visual Motion PhD Dissertation, University of Essex (1985)

10. Horn B.K.P. Robot Vision MIT Press, Cambridge, MA, USA (1986) 